\title{
Discord between morphological and phylogenetic species boundaries: incomplete lineage sorting and recombination results in fuzzy species boundaries in an asexual fungal pathogen
}

\author{
Jane E Stewart ${ }^{1,5^{*}}$, Lavern W Timmer ${ }^{2}$, Christopher B Lawrence ${ }^{3}$, Barry M Pryor ${ }^{4}$ and Tobin L Peever ${ }^{1}$
}

\begin{abstract}
Background: Traditional morphological and biological species concepts are difficult to apply to closely related, asexual taxa because of the lack of an active sexual phase and paucity of morphological characters. Phylogenetic species concepts such as genealogical concordance phylogenetic species recognition (GCPSR) have been extensively used; however, methods that incorporate gene tree uncertainty into species recognition may more accurately and objectively delineate species. Using a worldwide sample of Alternaria alternata sensu lato, causal agent of citrus brown spot, the evolutionary histories of four nuclear loci including an endo-polygalacturonase gene, two anonymous loci, and one microsatellite flanking region were estimated using the coalescent. Species boundaries were estimated using several approaches including those that incorporate uncertainty in gene genealogies when lineage sorting and non-reciprocal monophyly of gene trees is common.
\end{abstract}

Results: Coalescent analyses revealed three phylogenetic lineages strongly influenced by incomplete lineage sorting and recombination. Divergence of the citrus 2 lineage from the citrus 1 and citrus 3 lineages was supported at most loci. A consensus of species tree estimation methods supported two species of Alternaria causing citrus brown spot worldwide. Based on substitution rates at the endo-polygalacturonase locus, divergence of the citrus 2 and the 1 and 3 lineages was estimated to have occurred at least 5, 400 years before present, predating the human-mediated movement of citrus and associated pathogens out of SE Asia.

Conclusions: The number of Alternaria species identified as causing brown spot of citrus worldwide using morphological criteria has been overestimated. Little support was found for most of these morphospecies using quantitative species recognition approaches. Correct species delimitation of plant-pathogenic fungi is critical for understanding the evolution of pathogenicity, introductions of pathogens to new areas, and for regulating the movement of pathogens to enforce quarantines. This research shows that multilocus phylogenetic methods that allow for recombination and incomplete lineage sorting can be useful for the quantitative delimitation of asexual species that are morphologically indistinguishable. Two phylogenetic species of Alternaria were identified as causing citrus brown spot worldwide. Further research is needed to determine how these species were introduced worldwide, how they differ phenotypically and how these species are maintained.

Keywords: Coalescent, Species delimitation, Species tree, Gene tree

\footnotetext{
*Correspondence: janestew@uga.edu

'Department of Plant Pathology, Washington State University, Pullman, WA,

USA

${ }^{5}$ Current address: Department of Plant Pathology, University of Georgia,

Athens, Georgia

Full list of author information is available at the end of the article
}

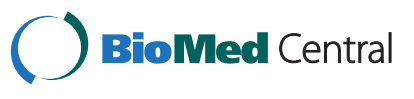

(c) 2014 Stewart et al.; licensee BioMed Central Ltd. This is an Open Access article distributed under the terms of the Creative Commons Attribution License (http://creativecommons.org/licenses/by/2.0), which permits unrestricted use, distribution, and reproduction in any medium, provided the original work is properly credited. The Creative Commons Public Domain Dedication waiver (http://creativecommons.org/publicdomain/zero/1.0/) applies to the data made available in this article, unless otherwise stated. 


\section{Background}

The delimitation of species and evolutionary relationships among them is fundamental to biology. However, the application of species concepts to putatively asexual taxa can be difficult and controversial $[1,2]$. Not all species concepts can be applied to asexual species, and some researchers have even suggested that asexual lineages do not represent species at all [3]. For example, the morphological species concept (MSC) or biological species concept (BSC) may not be adequate for delineating asexual fungi $[2,4,5]$ especially where morphological differences are not observed. However, any new allele conferring an adaptive advantage to an asexual organism in a particular ecological niche may be selected. Selective pressure on that gene is expected to affect the entire genome through genetic hitchhiking thus having the potential to rapidly form a new cryptic species [4]. These discrete entities may be recognized as species rather than as part of continuous distribution of phenotypes. Asexual taxa are also expected to diverge into discrete lineages under processes such as divergent selection and/or geographic isolation [4].

To date, the systematics of asexual fungi has relied heavily on phylogenetic approaches to study cryptic speciation among closely related taxa [5-7]. In asexual fungi, phylogenetic species concepts can identify phylogenetically distinct lineages with the implication that new species have formed that are not yet morphologically distinct [8]. These methods most often involve the concatenation of sequence alignments, using methods such as the genealogical concordance phylogenetic species recognition (GCPSR) which is an operational criterion for species recognition $[2,9]$. The GCPSR focuses on species identification through multi-gene genealogies and reciprocal monophyly to identify fungal species [5]. This method has applicability for both asexual and sexual lineages and species boundaries are estimated by concordant clades of multi-gene genealogies. The absence of monophyly and conflict among the multiple gene trees identifies species limits for taxa [5]. However, species boundaries of closely related taxa, in the initial stages of divergence, can be difficult to ascertain using multilocus phylogenetic methods because gene trees of recently diverged taxa can differ substantially in their evolutionary histories [10].

Processes such as incomplete lineage sorting, recombination, and horizontal transfer can cause discord among gene and species trees, masking true evolutionary relationships among closely related taxa [11]. Incongruence, in itself, can signal possible recombination, reticulation, and incomplete lineage sorting. Individual gene trees may have different evolutionary histories $[12,13]$ which limits the accuracy of species tree estimation using concatenation of loci [14]. Incomplete lineage sorting is caused when ancestral polymorphisms persist through speciation events and each ancestral polymorphism can lead to different alleles carried among descendants [14,15]. Coalescent-based methods, which stochastically join sampled gene lineages as they are followed back in time, have been developed to incorporate lineage sorting and the presence of incongruent genomic regions into phylogenetic estimation procedures [16-18], even in the presence of lineage sorting and lack of reciprocal monophyly at any single locus [10].

Coalescent methods have recently been used to assess species trees for a range of taxa including sexually reproducing species such as the Tennessee cave salamanders (Gyrinophilus; [19]), tropical lowland birds (Manacus; [20]), grasshoppers (Melanoplus; [10]), and rice [21]. Few researchers, however, have examined the utility of these methods for closely related asexual taxa that are morphologically indistinguishable [22]. Phylogenetic analyses of closely-related taxa, such as rice [21], Drosophila [23], and cryptic fungal species, such as Penicillium [22,24], are at the intersection of population genetics and phylogenetics where the effects of coalescent stochasticity results in high levels of gene tree incongruence [17,25-27]. Estimating species trees for these taxa can be problematic; these methods could prove to be useful for closely-related asexual fungal taxa.

The putatively asexual citrus pathogen, Alternaria alternata, provides an ideal case study for the application of quantitative species recognition using species tree estimation methods that incorporate uncertainty in gene trees. Andrew et al. [28] developed a species phylogeny for small-spored Alternaria using four genomic regions including a protein coding gene and three anonymous, non-coding regions. Significant incongruence was found among gene genealogies and several putative recombination events were identified within two of the non-coding regions indicating divergent evolutionary histories among the loci [28]. Hypotheses to explain this incongruence included recombination and incomplete lineage sorting. A recent study of the mating system of $A$. alternata causing brown spot in Florida found signatures of recombination [29] but studies of the larger worldwide population of the pathogen are lacking.

Currently, there is a large discord between the number of morphological and phylogenetic species that are thought to cause citrus brown spot. The fungus infects tangerines and mandarins (Citrus reticulata Blanco) and tangerine $\mathrm{x}$ grapefruit (C. reticulata $\mathrm{x} C$. paradisi Macfad.) hybrids worldwide. When first reported in Australia [30], the pathogen was identified as A. citri Ellis \& N. Pierce due to its morphological similarity to the causal agent of a postharvest disease, citrus black rot. Since this time, the pathogen has been referred to as A. alternata 'tangerine pathotype' based on morphological similarity to A. alternata [31-33]. Further, molecular comparisons were performed using restriction fragment length polymorphisms 
(RFLPs) of the nuclear ribosomal DNA (internal transcribed spacer region, ITS) among 11 closely related, small-spored Alternaria taxa. Results from this study showed that morphologically similar Alternaria species collected from different hosts and that produce host specific toxins shared common RFLP fingerprints and identical ITS sequences, leading the authors to conclude that Alternaria fungi known to produce host-specific toxins are intraspecific variants of $A$. alternata specialized in host pathogenicity [33].

Ten new Alternaria species have been described from citrus hosts [34] and phylogenetic studies have attempted to map these morphospecies onto phylogenies estimated from molecular data. Using a worldwide sample of isolates, Peever et al. [35,36] and Andrew et al. [28] evaluated these morphospecies using phylogenetic criteria and found three distinct lineages (labeled Clades 1, 2 and 3 in [35]). Two of these lineages were found in Florida (Clades 1 and 2), whereas the third lineage occurred only in Turkey, Israel, Australia and South Africa (Clade 3). These three worldwide lineages corresponded to several morphospecies including $A$. citriarbusti (Clade 1), A. tangelonis and A. colombiana (Clade 2), and A. dumosa, A. turkisafria, $A$. perangusta and A. interrupta (Clade 3) [34]. Peever et al. [36] further tested the concordance between the 10 citrusassociated morphospecies [34] on citrus using a broader range of isolates. Peever et al. [36] found eight distinct Alternaria clades from citrus hosts that could be interpreted as phylogenetic species under the GCPSR concept. This incongruence between the number of species defined using morphological and phylogenetic criteria raises significant questions about the number of Alternaria taxa that cause brown spot disease.

Coalescent analyses of gene geneaolgies, which describes descendent/ancestor relationships where the gene of interest undergoes coalescence to a common ancestor, can be used to examine the evolutionary history of a gene backwards in time and can be used to incorporate incomplete lineage sorting into phylogenetic analyses [37]. Currently, there is a lack of agreement among researchers about how many Alternaria species cause citrus brown spot, ranging from as many as 10 species to as little as 1 species. The main objective of this study was to quantitatively estimate the number of species of Alternaria causing citrus brown spot on a worldwide scale utilizing newly developed methods that incorporate the coalescent and account for recombination or incomplete lineage sorting. The evolutionary histories and recombination of known genetically distinct lineages, citrus 1 , citrus 2 and citrus 3 were evaluated from a larger worldwide sample of $A$. alternata tangerine pathotype and more loci in order to establish species boundaries of these previously observed phylogenetic lineages using gene sequence concatenation methods and several approaches that incorporate uncertainty in gene genealogies when lineage sorting and non-reciprocal monophyly of gene trees is common.

\section{Methods \\ Isolates}

One hundred and forty two isolates were collected from brown spot lesions on cultivars of tangerines, mandarins (Citrus reticulata Blanco) and tangerine $\mathrm{x}$ grapefruit hybrids [C. reticulata x C. paradise (Macfad.)] in 12 countries, including Argentina (AR), Australia (AU), Brazil (BZ), Colombia (CB), Greece (GR), Iran (IR), Israel (IS), Italy (IT), Peru (PE), Spain (SP), Turkey (TU), and USA (FL). Sixty-five of these isolates from Australia, Colombia, Israel, South Africa, and United States overlapped with a previous study ([35]; Figure 1A, Additional file 1: Table S1). Isolates from Argentina, Australia, Brazil, Colombia, Greece, Iran, Israel, Italy, Peru, Spain, Turkey, and USA were sampled from tangerine hybrids in several geographically separated citrus groves within each country. Australian isolates were collected from tangerine hybrids in the Narara arboretum (Narara, New South Wales), and isolates from Florida, USA were collected from a small area $\left(2,500 \mathrm{~m}^{2}\right)$ in a single grove of Minneola tangelo [35,36,38].

\section{DNA extraction}

Fungi were cultivated in potato dextrose broth (FisherSci, Pittsburg, PA) for 5-7 days at room temperature on an orbital shaker at $150 \mathrm{rpm}$. Genomic DNA was extracted from powdered, lyophilized mycelium following the methods of Peever et al. [38], using either a Qiagen DNeasy Kit or a Phenol-Chloroform procedure. Extracted DNA was quantified using a Nanodrop1000 (NanoDrop products, Wilmington, DE, USA), and a total of $30 \mathrm{ng}$ was used as template for PCR. Isolates were maintained in long-term storage on sterilized filter paper at $-20^{\circ} \mathrm{C}$ as previously described [38].

\section{Endo-polygalacturonase sequencing}

Each isolate was sequenced at the endo-polygalacturonase locus [39] using primers and conditions similar to those used by Peever et al. [35,36,40] and Andrew et al. [28]. This region has been extensively used because other commonly used genomic regions, such as ribosomal regions, mitochondrial large and small subunits, and the beta-tubulin gene, show little variation among Alternaria isolates collected from citrus [41]. Amplified products were visualized in $2 \%$ ethidium bromide-stained agarose gels. Amplified DNA fragments were sequenced directly on both strands following treatment with EXOSAP-IT (USB, Cleveland, $\mathrm{OH}$ ) using the Big Dye terminator kit (Applied Biosystems, Foster City, CA). Sequence reads were performed on either a PE Biosystems model 3700 automated DNA Sequencer by the Laboratory for Biotechnology and Bioinformatics at Washington State 
A

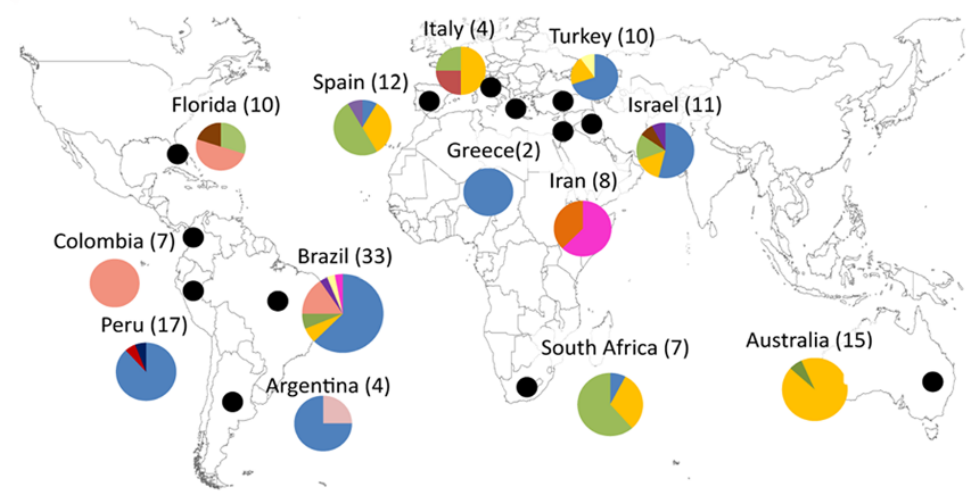

B

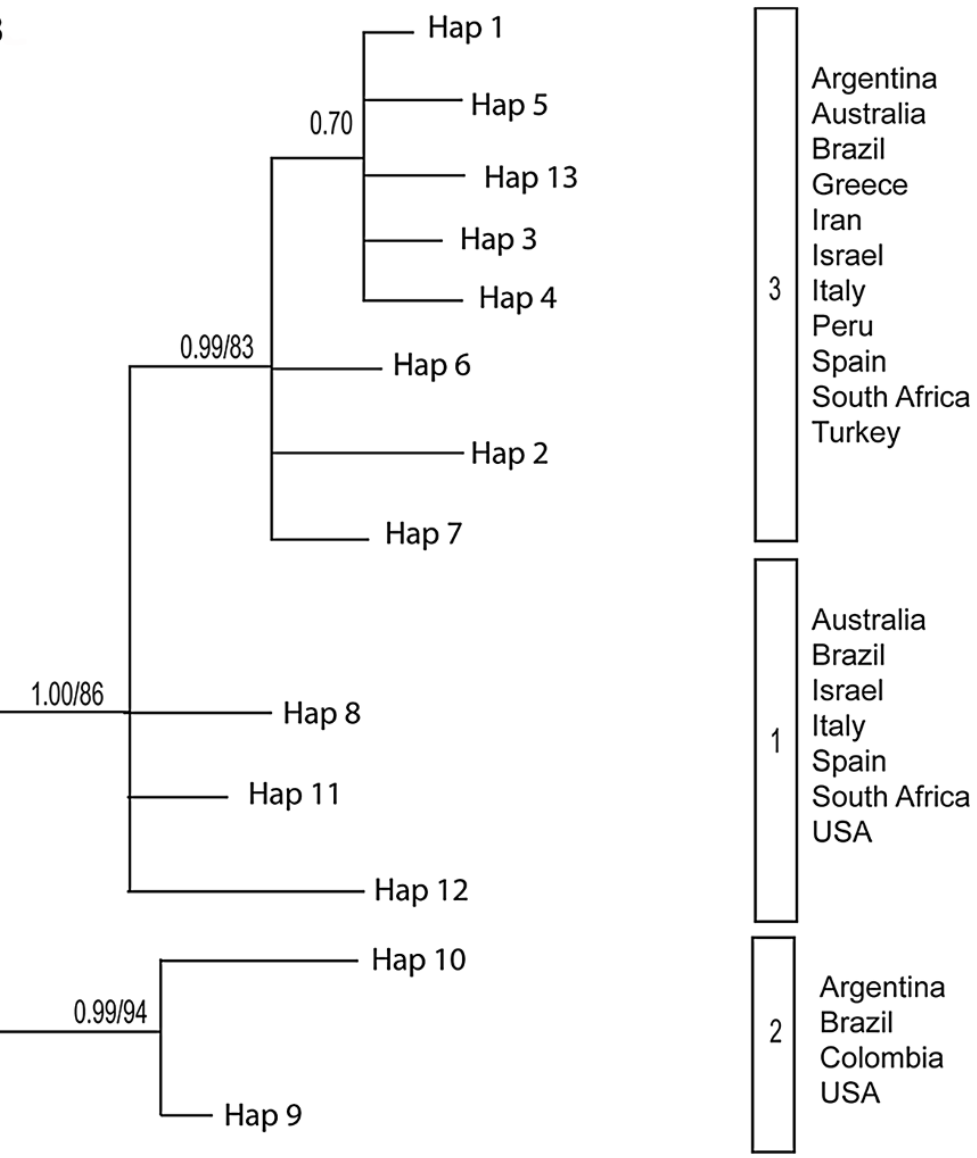

Alternaria tomato (BMP 2031)

0.001

Figure $1 \mathrm{~A}$. Geographic origin of Alternaria alternata sampled from citrus in 12 countries (small black circles). The numbers of isolates sampled from each country are in parentheses. The pie chart for each country represents the number of endoPG haplotypes with each color denoting a different endoPG haplotype [(Hap1 (Fuschia), Hap2 (orange), Hap3 (blue), Hap4 (dark blue), Hap5 (red), Hap6 (maroon), Hap7 (yellow), Hap8 (green), Hap9 (pink), Hap10 (light pink), Hap11 (brown), Hap12 (purple), and Hap13 (light yellow)]. B. An endo-polygalacturonase (endoPG) phylogeny estimated among a worldwide sample of citrus brown spot isolates using Bayesian inference with $A$. tomato as an outgroup. Three phylogenetic lineages identified correspond to Clades 1, 2, and 3 of Peever et al. [35]. Node support is given as posterior probabilities and bootstrap values based on Bayesian and likelihood analyses. 
University, Pullman, WA or at Elim Biopharmaceuticals, Inc, Wayward, CA.

\section{OPA1-3, OPA2-1, and Flank-F3 sequencing}

A subset of isolates with unique endoPG haplotypes representing each sampled location $(n=34)$ was selected for additional sequencing and analyses. Additional loci included two anonymous, non-coding SCAR markers OPA1-3 and OPA2-1 [28], and one non-coding microsatellite flanking region Flank-F3. Loci OPA1-3 and OPA2-1 were previously used for phylogenetic studies of small-spored A. alternata [36,42]. Amplification and sequencing conditions were as previously reported $[28,36,40,42]$. Clone sequences containing microsatellites [43] were downloaded from GenBank [accession: DQ272483 to DQ272487], and primers were designed to amplify the microsatellite flanking regions using Primer 3 [44]. Primer sequences for AA-Flank-F3 were (Flank3F-5'-AGCCAAAACACGTTGATACC-3'/ Flank3R5' ATCCGCAGCGAAAAGAACT-'3). Twenty microliter PCR reaction mixtures contained 20 ng genomic DNA, $1 \times$ PCR buffer (New England Biolabs (NEB), Ipswich, MA), $4 \mathrm{nmol}$ of each dNTP (NEB), 50 pmol primer, and $1 \mathrm{U}$ of Taq polymerase (NEB). Cycling conditions consisted of denaturation at $94^{\circ} \mathrm{C}$ for $4 \mathrm{~min}$; 44 cycles of $94^{\circ} \mathrm{C}$ for 1 min, $55^{\circ} \mathrm{C}$ for $30 \mathrm{sec}$, and $72^{\circ} \mathrm{C}$ for $2 \mathrm{~min}$; final extension was at $72^{\circ} \mathrm{C}$ for $7 \mathrm{~min}$ depending on the optimal conditions for each primer set.

\section{Phylogenetic analyses and congruence among loci}

Each locus was analyzed independently. Maximum likelihood and Bayesian phylogenetic analyses were performed for each locus using PhyML [45] and MrBayes 3.0 [46]. DT-ModSel [47] was used to estimate the nucleotide substitution models best representing each dataset. An Kimura K80 model was selected for the endoPG (base frequencies $=$ equal; transversion $=5.29$; proportion of variable sites $=0$ ), and OPA2-1 (base frequencies = equal; transversion ratio $=5.52$; proportion of variable sites $=0$ ). The Kimura K80 model with proportion of invariable sites was selected for OPA1-3 (base frequencies = equal; tratio $=1.47$; proportion of variable sites $=0$ ). The Jukes Cantor 69 model was selected for Flank-F3 (base frequencies = equal; proportion of variable sites $=0$ ). Maximum likelihood analyses were performed under the heuristic search with TBR branch-swapping, and bootstrap support was estimated using 1000 pseudoreplicates. For Bayesian analyses, Metropolis-coupled Markov chain Monte Carlo searches included 2 runs with four chains each run for 3,000,000 generations and ensuring that the average split frequencies between the runs was less than $1 \%$. Trees were sampled every 200 generations. Each run generated 60,001 trees of which the first 18,000 trees (30\% of the total number of generations) were discarded as "burnin", as visually determined by evaluating log files in TRACER version 1.5 [48].

In order to test topology congruence among phylogenies from different loci, the Shimodaira-Hasegawa $(\mathrm{SH})$ test of topological congruence [49] was conducted on the Bayesian phylogenies as implemented in PAUP"10_4b [50] with 1000 RELL resampling replicates.

\section{Coalescent analyses}

Ancestral histories of the citrus brown spot lineages were estimated using the coalescent $[37,45]$. Sequence data were aligned and edited manually by eye and using clustalW implemented in BioEdit v7.0.53 for Windows [51]. Isolates were assigned to haplotypes using DnaSP v $5.1[52,53]$. To verify the suitability of each locus for coalescent analyses, the neutrality of each locus was estimated using $\mathrm{Fu}$ and Li's D and Tajima's $D$ and potential recombination within each locus was examined using Rmin [54] as implemented in DnaSP. Incompatibility matrices [55] were estimated in SNAP Clade and SNAP Matrix as implemented in SNAP workbench [56] to visualize incompatible nucleotide sites, such as those arising from recombination or recurrent mutation. Sequences were collapsed into unique haplotypes using SNAP map [57] and SITES version 1.1 [58] by removing indels and incompatible sites.

Evolutionary histories were simulated using coalescent analyses for each locus. Two loci Flank-3 and OPA1-3 showed evidence for recombination and therefore coalescent analyses for all genomic regions were implemented using recom version 5.8 (within SNAP workbench), which allows for coalescent analyses with recombination, assuming the infinite-sites model, neutral evolution, panmixia and constant population size [59]. Using a haploid coalescent model, recom5.8 estimates population recombination rate, $\rho,\left(2 \mathrm{~N}_{\mathrm{e}} \mathrm{r}\right)$ and mutation rate, $\theta\left(2 \mathrm{~N}_{\mathrm{e}} \mu\right)$. These estimates were then used to obtain estimates of the number of recombination events and the time to the most recent common ancestor (TMRCA). For all analyses, the ancestral state of each segregating site was estimated by comparing each site to that of A. tomato isolate BMP2031 [60]. Sites which had ambiguous ancestral states were removed from the datasets. OPA1-3 had five sites $(205,230,301,382$, and 436$)$ with three states and one site (349) with four states, and the endoPG had one site (346) with three states. Coalescent analyses were performed in SNAP workbench [56], with five independent runs ( 1 million simulations each) per genomic region to ensure convergence for each parameter estimate. Conditions were switched to $+\mathrm{b}$ for genomic region OPA1-3, which aborts low probability paths and returns to zero. Gene geneaologies and minimal recombination graphs (ARGs) were constructed to graphically represent the evolutionary history of the citrus lineages estimated by the coalescent. No putative recombination 
events were detected within endoPG and OPA2-1. Genetree [61], which assumes coalescent analyses without recombination, was used to estimate the coalescent gene genealogies for these regions and compared to the recom5.8 results. Five independent simulations with 1 million runs each were conducted on haplotypes of each region to estimate the ages of mutations and the TMRCA, as inferred by an $A$. tomato rooted tree, to assess convergence. A graph of the tree was generated with coalescent unit times using Treepic [62]. An ancestral recombination graph (ARG) can be used to visualize a recombining coalescent history that cannot be displayed using a bifurcating tree [63]. The ancestral history of the recombining OPA1-3 and Flank-F3 haplotypes were reconstructed using a parsimony approach that accounts for both mutation and recombination backwards in time. Beagle [63] was used to produce an ARG for Flank-F3, whereas kwarg was used for OPA1-3 [63]. Beagle computes minimum recombination histories with an exhaustive approach. Kwarg, on the other hand, implements a heuristic search for plausible histories and does not guarantee the minimal recombination history. Beagle was run for both genomic regions, however due to the increased complexity and putative number of recombination events within OPA1-3, each trial run crashed.

\section{Species tree estimation}

Four methods were used to estimate species trees among the four gene trees. These included concatenation, genealogical concordance phylogenetic species recognition (GCPSR; [5]), minimizing deep coalescence (MDC; $[14,17,64])$, and a mixture of coalescent and the Yule process ("BEAST) [48]. For MDC and "BEAST, taxa are required to be assigned to species a priori. Therefore, taxa were assigned to species based on identified endoPG clades (citrus 1, citrus 2, and citrus 3, Additional file 1: Table S1).

A phylogeny of the concatenated dataset was implemented in MrBayes. Loci were partitioned and the previously estimated evolutionary models were used for Bayesian analyses. Metropolis-coupled Markov chain Monte Carlo searches included 2 runs with four chains each run for 3,000,000 generations and ensuring that the average split frequencies between the runs was less than $1 \%$. Each run generated 60,001 trees of which the first 18,000 trees (30\% of the total number of generations) were discarded as "burnin".

GCPSR identifies species boundaries by comparing multiple gene trees among the same set of taxa $[5,65]$. Putative species are identified when representatives of a species formed well-supported clades (95 posterior probability/70 bootstrap) in all gene trees [66]. Bayesian and likelihood tree searches were used to estimate species within the citrus brown spot worldwide population according to GCPSR criteria.

The MDC approach assumes that discordance of gene trees is the result of incomplete lineage sorting $[14,17]$.
The deep coalescence measure is a count of the number of extra gene lineages that result from fitting a gene tree into a species tree, thereby summing the extra gene lineages as a measure of discordance. MDC then searches for a species tree by minimizing the number of deep coalescences across loci. MDC analysis was implemented in Mesquite v2.73 [67]. Gene tree uncertainty was accommodated into species tree inference by resampling ( 500 times) the posterior probability of tree topologies obtained from the Bayesian phylogenetic analyses using the Mesquite software module AUGIST [64]. To fit the gene trees into a species tree that minimized the number of deep coalescence across loci, gene trees were considered rooted (A. tomato as the root) and a heuristic search utilizing subtree pruning and re-grafting was used. All equally parsimonious species trees were retained, and a 50\% majority-rule consensus tree was generated. Biparition frequencies for nodes were used as measures of species tree uncertainty.

"BEAST generates posterior samples by simultaneously estimating gene and species trees under a hierarchical coalescent model while allowing for independent evolutionary processes in each genomic region. BEAUTi version 1.7.5 [48] was used to create XML-formatted input files for "BEAST v1.7.5. Substitution models were chosen as previously described and were unlinked across genes with parameters estimated separately for each gene. As a needed prior in "BEAST, isolates were assigned to species groups under the Traits tab based on the three clades identified in the endoPG phylogeny. Evolutionary rates were estimated under a Yule process [68]. A Yule model was chosen as the species tree prior, which assumes a constant lineage birth rate for each branch in the tree. This tree prior is most suitable for trees describing the relationships between individuals from different species and is often thought of as describing the net rate of speciation [69]. Species tree estimations were carried out based on strict molecular clock assumption, following the methods of Heled and Drummond [48]. Data sets were run for 50 million generations in BEAST, sampling every 5,000 generations. Analyses were performed twice. Postburnin trees were combined with the program LogCombiner (BEAST v 1.6.0), and chains were assumed to converge when the average standard deviation of split frequencies was found to be $<0.011$. The maximum clade credibility tree with posterior probability of each node was computed with the program TreeAnnotator (BEAST v 1.6.0). Log files were evaluated in TRACER version 1.5 [48]. The species tree was calculated using TreeAnnotator version 1.7.2 with a burn-in of 5000 trees. FigTree version 1.3.1 [70] was used to visualize the consensus tree node ages, branch lengths and posterior probabilities. 


\section{Results}

\section{Worldwide phylogenetic lineages}

Alternaria alternata sampled from tangerine and tangerine hybrids on a worldwide scale $(n=142)$ revealed a total of 13 endoPG haplotypes (Additional file 1: Table S1, Figure 1A). Bayesian and maximum likelihood analyses of endoPG resulted in two phylogenetic lineages using a 95 posterior probability and 70 bootstrap value criterion (Figure 1B) [66]. These two lineages are subsequently referred to as 'citrus 2' and 'citrus 3'. All other haplotypes (Hap8, Hap11, and Hap12) were placed into another group called citrus 1 based on previously published results that showed the existence of three lineages [35].

\section{Coalesence analyses}

Thirty-five representative isolates were selected based on unique endoPG haplotype and geographical location to resolve the ancestral histories of the lineages using coalescent-based approaches. Neutrality and intra-locus recombination rates were estimated for all the genomic regions using D (Tajima's and Fu and Li's) statistics and Rmin. We were unable to reject the null hypothesis of neutrality for two loci (Table 1).

Coalescent-derived gene genealogies were estimated for the two non-recombining loci, endoPG and OPA2-1 (Figures 2A-B). In the endoPG genealogy, isolates of the citrus 1 lineage were positioned ancestral to all other haplotypes by several mutations (Figure 2A). In locus OPA2-1 signals of incomplete lineage sorting, where ancestral polymorphisms have not been sorted, were evident (Figure 2B). For example, some isolates from citrus 1 and citrus 3 were interspersed among identified clades, suggestive of non-reciprocal monophyly (Figure 2B). Further, little variation was revealed in the OPA2-1 genealogy, but eleven mutations, respectively, separated the $A$. alternata lineages from the $A$. tomato outgroup.
Isolates from all lineages shared haplotypes, such that isolates from lineages citrus 1 and 3 formed a paraphyletic group. This was also observed in the OPA2-1, where haplotypes D and $\mathrm{G}$ included isolates from lineages citrus 1 and 3. Two clusters were formed, one including isolates from citrus 1 and 3, while the other included a polytomy of haplotypes from the citrus 1 and citrus 2 lineages (Figure 2B).

Loci OPA1-3 and Flank-F3 had evolutionary histories of recombination. Three recombination events were observed in Flank-F3, which in all cases involved isolates from lineage citrus 1 and 2 (Figure 3A). OPA1-3 had the most complex evolutionary history involving eight recombination events (Figure 3B). These events included isolates from all three lineages suggesting possibly a sexual past as suggested by Berbee et al. [71].

\section{Phylogenetic analyses}

Analyses were conducted for each locus independently for 35 isolates with representative endoPG haplotypes selected from the original worldwide sample. Analysis of endoPG yielded a phylogeny with similar topology to that estimated among the total worldwide sample, supporting three clades (Figure 4A). Eight haplotypes were identified in locus OPA2-1, however no monophyly was observed in the lineages (Figure 4B). A total of 7 haplotypes were identified locus Flank-3 (Figure 4C). Only one well-supported clade was found, including all isolates from citrus 2. Locus OPA1-3, with 13 haplotypes, had the most phylogenetic resolution, resulting in six well-supported clades (Figure 4D). Among 503 sites, 75 sites $(14.7 \%)$ were polymorphic. Of these, 55 (10.9\%) were parsimony informative (Table 1). Of these clades, one corresponded to a terminal clade including all citrus 2 haplotypes, clearly separated from but sister to one citrus 1 haplotype, and polyphyletic relationships were observed in lineages 1 and 3 (Figures 4A-D).

Table 1 Summary statistics of DNA polymorphisms in a worldwide sample of brown spot pathogen using four loci

\begin{tabular}{|c|c|c|c|c|c|c|}
\hline Locus (No. of isolates) & endoPG $(142)^{\mathrm{W}}$ & endoPG (35) & OPA2-1 (35) & F3 (35) & OPA1-3 (35) & Combined (35) \\
\hline No. of sites $\left({ }^{A}\right)$ & $427(422)$ & $427(421)$ & $538(535)$ & $222(220)$ & $503(493)$ & $1672(1686)$ \\
\hline No. of poly. sites $\left(\mathrm{PI} / \mathrm{PUI} \mathrm{I}^{\mathrm{B}}\right)$ & $21(10 / 11)$ & $24(8 / 16)$ & $20(14 / 6)$ & $15(8 / 7)$ & $75(55 / 20)$ & $135(77 / 58)$ \\
\hline No. of haplotypes ${ }^{c}$ & 13 & 13 & 7 & 7 & 12 & 24 \\
\hline Haplotype diversity & 0.72 & 0.86 & 0.80 & 0.48 & 0.87 & 0.95 \\
\hline Nucleotide diversity & 0.007 & 0.009 & 0.004 & 0.009 & 0.041 & 0.017 \\
\hline Tajima's D & $-0.78^{\mathrm{NS}}$ & $-1.10^{\mathrm{NS}}$ & $-1.70^{\mathrm{NS}}$ & $-1.35^{\mathrm{NS}}$ & $0.06^{\mathrm{NS}}$ & $-0.62^{N S}$ \\
\hline Fu and Li's D & $-4.05^{\mathrm{S}}$ & $-2.99^{\mathrm{S}}$ & $-2.96^{\mathrm{S}}$ & $-1.60^{\mathrm{NS}}$ & $-0.16^{\mathrm{NS}}$ & $-1.47^{\mathrm{NS}}$ \\
\hline Rec events $^{R}$ & N/A & 0 & 0 & 6 & 8 & $N / A$ \\
\hline
\end{tabular}

${ }^{\mathrm{A} E x c l u d i n g}$ indels.

${ }^{B}$ Number of polymorphic sites (informative/uninformative)

CExcludes outgroup isolate, BMP2031 (A. tomato).

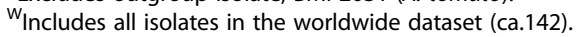

${ }^{\text {NS Non-significant, }}{ }^{\text {S}}$ Significant; $P>0.10$.

${ }^{\mathrm{R}}$ Number of recombination events estimated by Recom v. 5.8 [59]. 


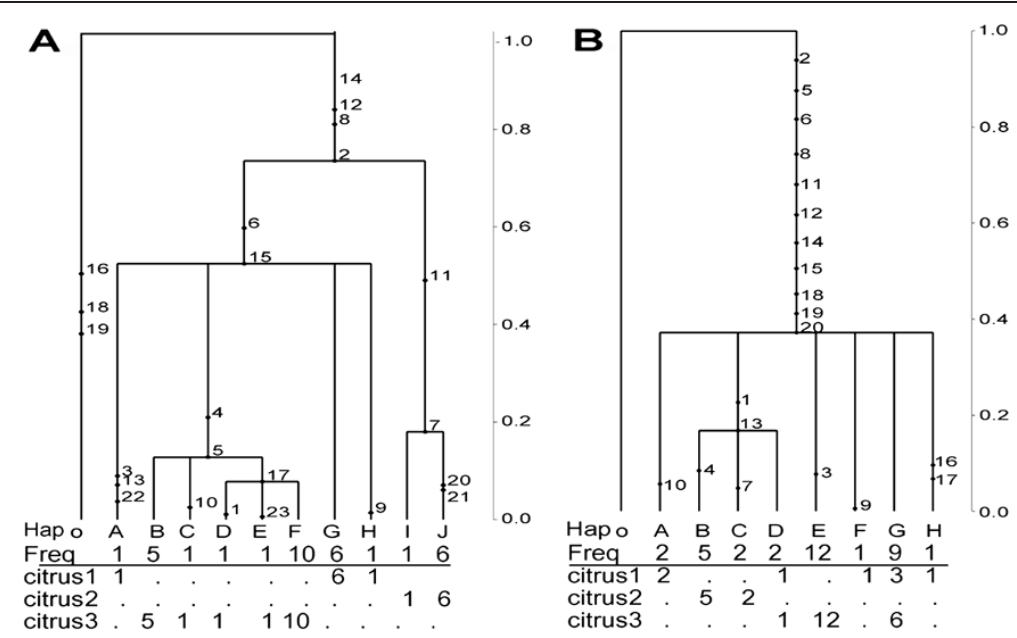

Figure 2 Coalescent-based gene genealogies estimated assuming no recombination for loci (A) EndoPG and (B) OPA2-1, with no conflicting sites. Each genealogy is scaled to TMRCA of 1 and numbers represent mutations. Each haplotype is labeled with a letter and the frequencies of each haplotype and relationship to each lineage is shown.

\section{Divergence time estimation for the endoPG}

A total of six synonymous nucleotide changes were observed when comparing haplotypes of the citrus lineages 1 and 3 against haplotypes from citrus 2 . Though only a portion of the CDS region was analyzed (427 out of $1137 \mathrm{bp}$ ), using the published rates for neutral gene substitution rate of $0.9 \times 10^{-9}$ and $16.7 \times 10^{-9}$ [72] resulted in a divergence date of at least 5,400 thousand years before present.

\section{Estimation of species trees from phylogenetically incongruent gene genealogies}

In the phylogenies from the concatenated and individual datasets, lineages citrus 1 and 3 were polyphyletic. Support for the citrus 2 lineage was found in most gene trees when GCPSR criteria was applied (Figure 4A-D). The citrus 1 and 3 lineages were only well-supported in the endoPG phylogeny and thus constituted a single, polyphyletic species when the other loci were considered.

Congruence of tree topology was tested and statistically significant incongruence was detected for all pairwise comparisons of all loci with OPA1-3 $(P=0.00)$ using Shimodaira-Hasegawa tests (Table 2). All other pairwise comparisons were non-significant.

The phylogeny generated from the concatenated dataset yielded four well-supported clades with topology similar to the endoPG phylogeny (Figure 5). The citrus 1 and 3 lineages were again polyphyletic, with individuals from each falling into three clades. However, lineage citrus 2 separated into one monophyletic well-supported (1.00 posterior probability) lineage.

Little support for multiple species was obtained using the MDC approach. In the MDC-estimated species tree, all lineages clustered into one clade with high bi-partition frequency (over 98\%) (Figure 6A). In contrast, "BEAST analyses revealed two well-supported species, citrus 2 and a second species comprising lineages citrus 1 and 3 (Figure 6B).

\section{Discussion}

Coalescent-based approaches and ancestral recombination graphs were used to elucidate the evolutionary history of the citrus brown spot pathogen on a worldwide scale and quantitatively estimate the number of species causing this disease. The evolutionary history of these lineages showed patterns of incomplete lineage sorting and recombination as has been observed among other closely-related taxa $[10,14]$ and was hinted at in previous phylogenetic studies of Alternaria [42]. Lineage sorting, recombination, and horizontal transfer [17] make phylogenetic analyses and species delimitation among small-spored Alternaria challenging $[28,36,40]$. In contrast to previous studies that described ten morphospecies causing Alternaria brown spot of citrus [34] we identified one or two species among three phylogenetic lineages using the "minimize deep coalescence" (MDC) and hierarchical Bayesian model approaches, respectively. Both methods failed to differentiate the citrus 1 and 3 lineages but the hierarchical Bayesian model method differentiated the citrus 2 lineage from citrus 1 and 3. Species boundaries between the morphospecies A. citriarbusti (citrus 1) and A. dumosa, A. turkisafria, $A$. perangusta and $A$. interrupta (citrus 3 ) were poorly supported by both methods. However, the GCPSR and *BEAST analyses separated the morphospecies A. tangelonis and A. colombiana (citrus 2 ) from citrus 1 and 3 . The hypothesis that all host-specific toxin producing, small-spored Alternaria taxa infecting citrus as well as other hosts such as strawberry, Japanese pear, apple, and tomato represent sub-specific variants or "pathotypes" of $A$. alternata is not 


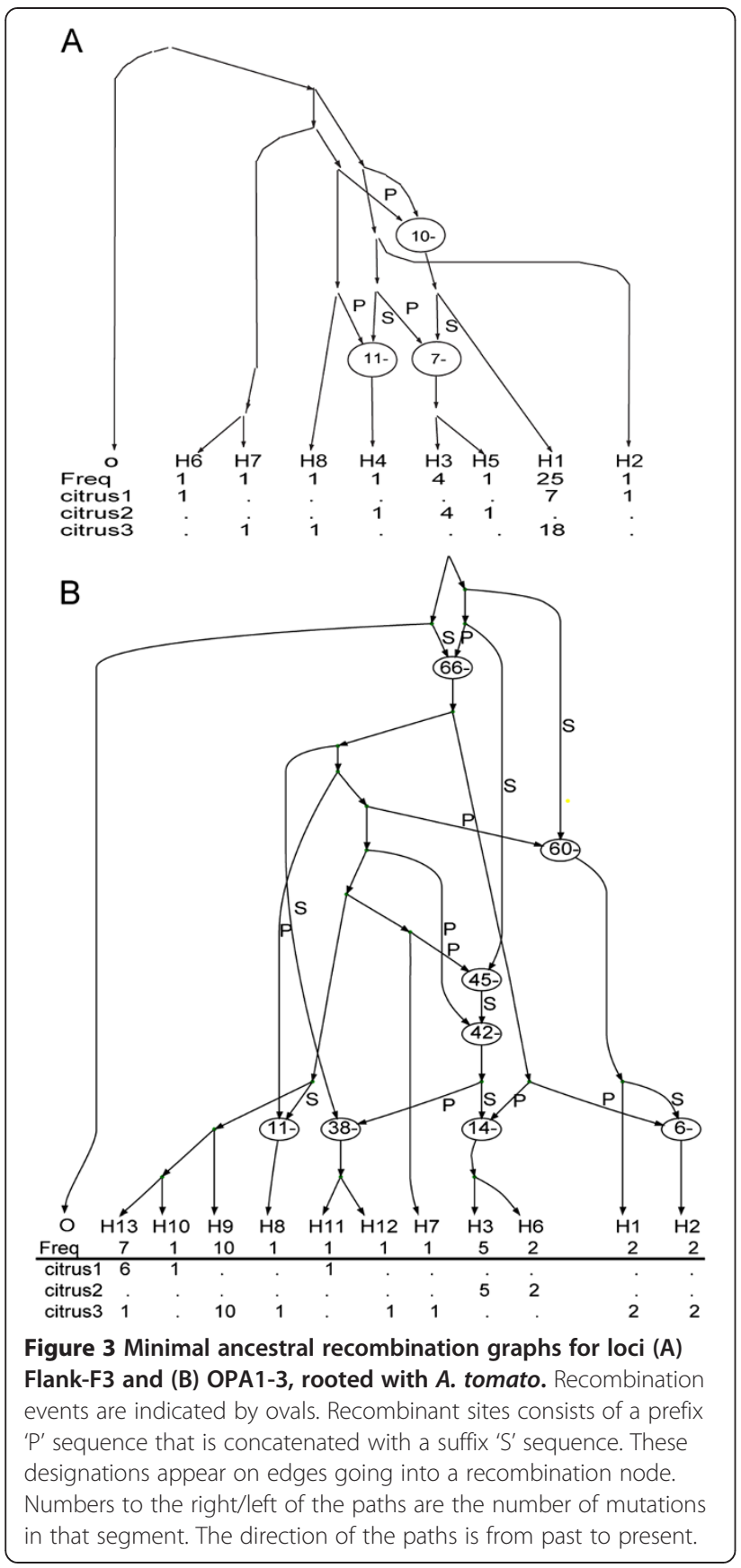

new [73]. One of our analyses supported this hypothesis and all other analyses conclusively demonstrated that the number of taxa causing brown spot disease of citrus has been over-estimated using morphological criteria. Further study is required to determine if the citrus 2 lineage represents a distinct species, and if so, what these two species should be named.

\section{Evolution of the citrus pathogens}

Alternaria alternata is an interesting model with which to study speciation in putatively asexual taxa. Asexual taxa do not easily fit within species concepts developed for sexual taxa $[2,4,8,74,75])$. In addition to being a wellrecognized saprotroph, $A$. alternata infects a wide range of hosts, including citrus, pear, strawberry, and apple $[73,76,77]$. Pathogenic forms of A. alternata are thought to have radiated from a recent common saprophytic ancestor through the horizontal acquisition of pathogenicity factors $[73,78]$. Isolates sampled for this study are considered representative of the "tangerine pathotype" $[38,79,80]$ and these fungi produce host-specific ACTtoxins that are required for pathogenicity $[74,81]$. ACTtoxins are structurally similar to the host-specific toxins produced by strawberry pathotype (AFT-toxins) and Japanese pear pathotype isolates (AKT-toxins) [81] and the genes controlling the biosynthesis of these toxins are homologous $[81,82]$ Connecting the three citrus lineages to the lineages of strawberry and Japanese pear pathotype isolates in future studies will allow polarization of the phylogeny of host-specific toxin-producing Alternaria and facilitate studies of toxin gene evolution and the evolution of pathogenicity. Assuming that the mutation rate for the endoPG gene is similar to other protein coding genes at approximately $0.9 \times 10^{-9}$ to $16.7 \times 10^{-9}$ mutations per site per year $[71,72,83]$ the estimated time of divergence of the citrus 2 lineage and the citrus 1 and 3 lineages is at least 5, 400 years before present, and probably occurred much earlier. This date suggests that divergence of citrus 2 occurred long before the movement of citrus, and presumably its pathogens, from its putative center of origin in southeastern Asia less than 600 years ago [84]. To date, only citrus 2 isolates have been isolated from North and South America, whereas the other lineages are found on several Old and New World continents. This suggests that independent introductions of each lineage may have occurred in different locations, presumably with the host.

Using GCPSR criteria, many cryptic species have been identified, including several plant pathogenic fungi [85-87], human-infecting fungi [88-90], and an insect pathogen [11]. Latin names have been assigned to some of these cryptic species [91-93]. As far as we are aware, this is the first report that compares the GCPSR method to other newly developed tree species estimation methods. Our results suggest that in the presence of divergent evolutionary histories, GCPSR will likely overestimate the number of species. Although no reports could be found comparing the GCPSR method with others, several studies have compared species tree estimates generated with "BEAST and/or MDC to concatenated results. It is now well-documented that concatenated data can produce well-supported phylogenies that are inconsistent with the true species tree [13,94-96]. Furthermore, Belfiore et al. [97] developed species trees using concatenation and BEST (Bayesian 


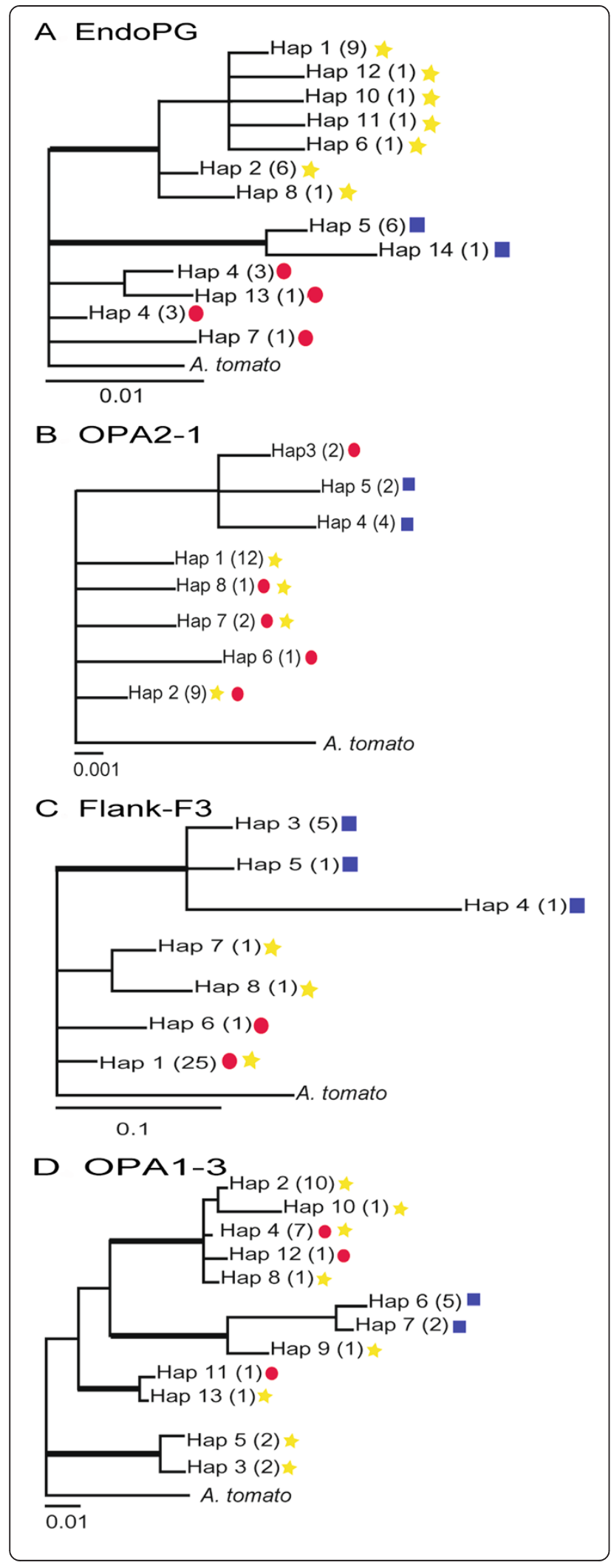

Figure 4 Bayesian phylogenies estimated for each locus, (A) endoPG, (B) OPA2-1, (C) Flank-F3, and (D) OPA1-3. Bold branches highlight nodes that were supported by $70 \%$ bootstrap values (maximum likelihood, 1000 replications) and 95 posterior probabilities. Numbers in parentheses represent the number of isolates of each haplotype. EndoPG haplotypes sampled from the citrus 1, citrus 2 and citrus 3 phylogenetic lineages are indicated by red circles, blue squares, and yellow stars, respectively.

Estimation of Species Tree) for pocket gophers of the genus Thomomys and found that the concatenated tree estimated from seven loci was over-resolved whereas fewer species were supported in the phylogeny estimated using BEST [97]. This result is similar to what we observed in this study with $A$. alternata. Further, Eckert and Carstens [11] tested the accuracy of concatenation and MDC generated species trees using simulated datasets in the presence of gene flow. As the level of gene flow increased the probability of identifying the true species using concatenation dropped to zero, although this value only decreased to $74 \%$ using MDC [11]. We found similar results, with the "BEAST and concatenated trees having similar topologies, but with node support varying widely. Our results suggest that species tree estimation methods that account for gene tree uncertainty among loci with diverged histories, with signals of lineage sorting and recombination may result in fewer well-supported species than concatenation, especially among closely related fungal taxa.

Two of the loci we employed in this study (Flank-F3 and OPA1-3) revealed strong evidence for a history of recombination. Asexual Alternaria species are thought to be derived from sexual ancestors [71], as has been suggested for other asexual ascomycetes [98], and one species $A$. infectoria has been connected to a Lewia teleomorph [34]. Although time scales for our ancestral recombination graphs (ARG) are not possible, the ARG for Flank-F3 showed three possible recombination events where citrus 2 haplotypes are possibly derived from citrus lineages 1 and 3 . It is difficult to date these putative recombination events and to know if these events are the result of historic or current sexual or asexual recombination. Signatures of recombination were found in the citrus 1 lineage in an Alternaria brown spot fungus

Table 2 Pairwise Shimodaira-Hasegawa tests of topological congruence among phylogenies

\begin{tabular}{lccc}
\hline & OPA2-1 & OPA1-3 & Flank-F3 \\
\hline endoPG & $0.059^{a}$ & 0.000 & 0.063 \\
OPA2-1 & & 0.000 & 0.128 \\
OPA1-3 & & & 0.000
\end{tabular}

aprobability of different topologies among 2000 bootstrapped datasets generated using RELL sampling. 


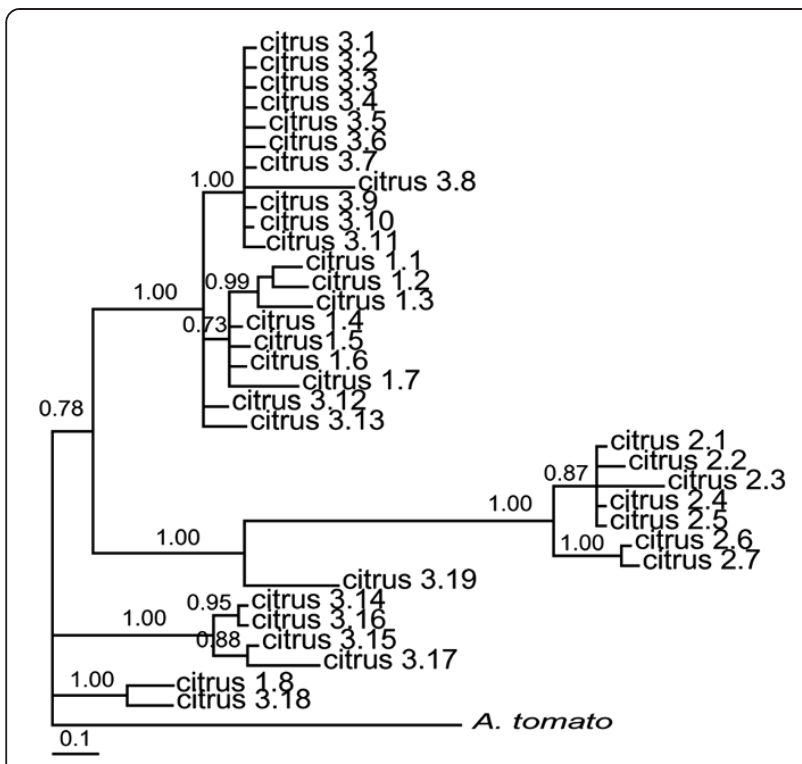

Figure 5 Bayesian phylogeny derived from the concatenated dataset with a total of 1672 nucleotides from four loci including the endo-polygalacturonase gene, SCAR markers OPA2-1 and OPA1-3, and one microsatellite flanking region AA-Flank-3. Node support in posterior probability is indicated for each node.

population in Florida [29] but the mechanism of this recombination is not known. Alternaria may be able to recombine through parasexual and/or sexual means [29], which concurs with the findings that mating type genes in A. alternata are expressed [5] and under strong purifying selection [99]. Further studies will be needed to determine if Alternaria alternata sensu lato is capable of forming a sexual stage than has heretofore been overlooked. If so, results from our ARG may represent contemporary rather than historical recombination, although dating recombination events in the ARG is not possible.

\section{Conclusions}

Species delimitation is important for the study of the evolution of pathogenicity and the emergence of infectious diseases. Further, the delimitation of species also plays a critical role in global biosecurity by providing guidelines for restrictions on the movement of plant pathogens among countries which has national and international significance $[100,101]$. The threat of movement of introduced pathogens around the world has resulted in the quarantine of many crops or the rejection of exported crops. Incorrectly naming a new species or wrongly identifying a species can result in significant economic losses [102]. In 2001-2002, shipments of Li Ya pear imported from China were rejected because of signs of Alternaria spp. infection, which also occurred in Australia, New Zealand, and United Kingdom. Two related species, A. alternata and A. gaisen, were included in a pest risk assessment and further research

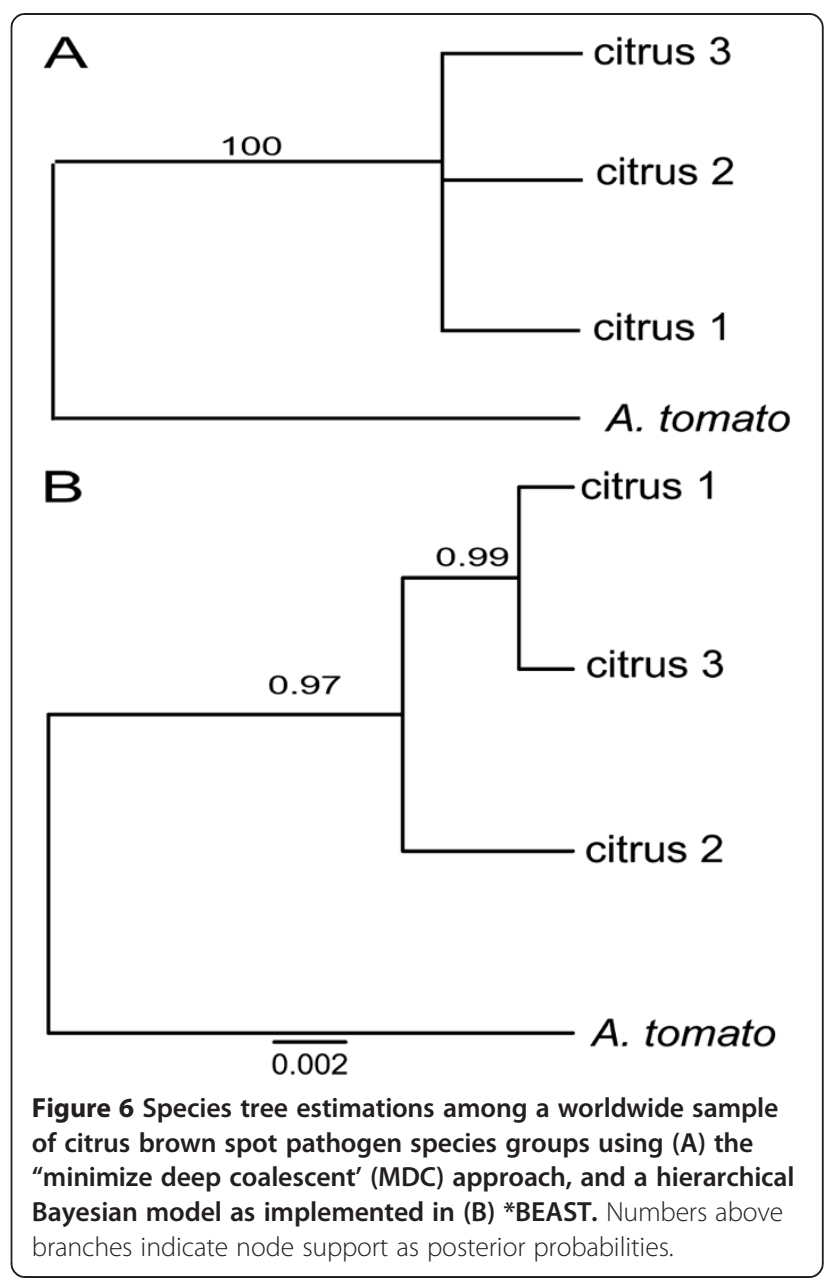

showed that isolates sampled from Li Ya pear were morphologically distinct and given a new name, A. yaliinficiens [100]. Our results indicate that citrus brown spot is caused by a maximum of two species of Alternaria, and that taxonomic revision of Alternaria infecting citrus, based on congruent morphological and genetic analyses, is needed. One of these species (encompassing lineages citrus 1 and citrus 3 ) is found worldwide but the second (lineage citrus 2) species has only been found in the Americas. It is not yet known if phenotypic differences in aggressiveness, host range, or growth rates exist between these species, but if so, limiting the movement of the citrus 2 species into new countries might be warranted. This study highlights the need for the use of these new methods to accurately identify closely related, morphologically indistinguishable species that are important in agriculture and potentially of regulatory interest.

\section{Availability of supporting data}

The data sets supporting the results of this article are available in GenBank under accession numbers KF699389-KF699527. 


\section{Additional file}

Additional file 1: Table S1. Isolates used in the study [103-105].

\section{Competing interests}

The authors declare that they have no competing interests.

\section{Authors' contributions}

JES and TLP conceived of the study. JES carried out molecular genetic studies and bioinformatics, and analyses. JES, TLP, LPT, CML, and BMP wrote and edited the manuscript. All authors read and approved the final manuscript.

\section{Acknowledgements}

We thank Dr. Marion Andrew, Dr. Francesca Rotondo and Kalyn A. Thomas for sequencing assistance. We also thank Dr. Zaid Abdo for critically reading and for comments that vastly improved this manuscript, and Dr. Hilal Özkilinç for fruitful discussions. This research was funded by NSF DEB0918668.

\section{Author details}

${ }^{1}$ Department of Plant Pathology, Washington State University, Pullman, WA, USA. ${ }^{2}$ Citrus Research and Education Center, University of Florida, Lake Alfred, FL, USA. ${ }^{3}$ Virginia Bioinformatics Institute, Blacksburg, VA, USA. ${ }^{4}$ Division of Plant Pathology and Microbiology, School of Plant Sciences, University of Arizona, Tucson, AZ, USA. ${ }^{5}$ Current address: Department of Plant Pathology, University of Georgia, Athens, Georgia.

\section{Received: 9 October 2013 Accepted: 21 February 2014} Published: 3 March 2014

\section{References}

1. Perkins DD: In Praise of Diversity. In More Gene Manipulations in Fungi. Edited by Bennett JW, Lasure LL. New York: Academic Press; 1991:3-26.

2. Harrington TC, Rizzo DM: Defining species in the fungi. In Structure and Dynamics of Fungal Populations. Edited by Worrall JJ. Dordrecht, The Netherlands: Kluwer Press; 1999:43-71.

3. Maynard Smith J, Szathmáry E: The Major Transitions in Evolution. London: Oxford University Press; 1995

4. Kohn LM: Mechanisms of fungal speciation. Annu Rev Phytopathol 2005, 43:279-308

5. Taylor JW, Jacobson DJ, Kroken S, Kasuga T, Geiser DM, Hibbett DS, Fisher MC: Phylogenetic species recognition and species concepts in fungi. Fungal Genet Biol 2000, 31:21-32.

6. Hibbett DS, Taylor JW: Fungal systematics: is a new age of enlightenment at hand? Nat Rev Microbiol 2013, 11:129-133.

7. Hawsworth DL: Integrating Morphological and Molecular Data in Fungal Systematics. In Systematics and Evolution of Fungi. Edited by Misra JK, Tewari JP, Deshmukh SK. New York, NY: CRC Press; 2012:1-14.

8. Kohn LM: The clonal dynamic in wild and agricultural plant populations. Can J Bot 1995, 73:S1231-S1240.

9. Taylor JW, Jacobson DJ, Fisher MC: The evolution of asexual fungi: reproduction, speciation, and classification. Annu Rev Phytopathol 1999, 37:197-246.

10. Carstens BC, Knowles LL: Estimating species phylogeny from gene-tree probabilities despite incomplete lineage sorting: An example from Melanoplus grasshoppers. Syst Biol 2007, 56:400-411.

11. Eckert AJ, Carstens BC: Does gene flow destroy phylogenetic signal? The performance of three methods for estimating species phylogeneies in the presence of gene flow. Mol Phylogenet Evol 2008, 49:832-842.

12. Kubatko LS, Carstens BC, Knowles LL: STEM: species tree estimation using maximum likelihood for gene trees under coalescence. Bioinformatics 2009, 25:9-973.

13. Kubatko LS, Degnan JH: Inconsistency of phylogenetic estimates from concatenated data under coalescence. Syst Biol 2007, 56:17-24.

14. Maddison WP: Gene trees in species trees. Syst Biol 1997, 46:523-536.

15. Hartl DL, Clark AG: Principles of Population Genetics. 4th edition. Sinauer Associates, Inc: Sunderland, MA; 2007.

16. Degnan JH, Salter LM: Gene tree distributions under the coalescent process. Evolution 2005, 9:24-37.
17. Maddison WP, Knowles LL: Inferring phylogengy despite incomplete lineage sorting. Syst Biol 2006, 55:410-431.

18. Lui L, Pearl DK: Species trees from gene trees: Reconstructing Bayesian posterior distributions of a species phylogeny using estimated gene tree distributions. Syst Biol 2007, 56:504-514.

19. Niemiller ML, Fitzpatrick BM, Miller BT: Recent divergence with gene flow in Tennessee cave salamanders (Plethodontidae: Gyrinophilus) inferred from gene genealogies. Mol Ecol 2008, 17:2258-2275.

20. Brumfield RT, Liu L, Lum DE, Edwards SV: Comparison of species tree methods for reconstructing the phylogeny of bearded manikins (Aves: Pipridae: Manacus) from multilocus sequence data. Syst Biol 2008, 57:9-731.

21. Cranston KA, Hurwitz B, Ware D, Stein L, Wing RA: Species trees from highly incongruent gene trees in rice. Syst Biol 2009, 58:489-500.

22. Henk DA, Eagle CE, Brown K, VanDenBerg MA, Dyer PS, Peterson SW, Fishe MC: Speciation despite globally overlapping distributions in Penicillium chyrysogenum: the population genetics of Alexander Fleming's lucky fungus. Mol Ecol 2011, 20:4288-4301.

23. Pollard DA, lyer VN, Moses AM, Eisen MB: Widespread discordance of gene trees with species tree in Drosophila: evidence for incomplete lineage sorting. PLoS Genet 2006, 2:e173.

24. Browne AGP, Fisher MC, Henk DA: Species-specific PCR to describe local-scale distribution of four cryptic species in the Penicillium chrysogenum complex. Fungal Ecol 2013, 6:419-429.

25. Pamilo P, Nei M: Relationships between gene trees and species trees. Mol Biol Evol 1988, 5:568-583.

26. Leache AD: Species tree discordance traces to phylogeographic clade boundaries in North American fence lizards (Sceloporus). Syst Biol 2008, 56:547-559.

27. Linnen CR: Species tree estimation for complex divergence histories: a case study in Neodiprion sawflies. In Estimating Species Trees. Edited by Knowles LL, Kubatko L. New York: Wiley-Blackwell; 2010.

28. Andrew M, Peever TL, Pryor BM: An expanded multilocus phylogeny does not resolve morphological species within the small-spored Alternaria species complex. Mycologia 2009, 101:95-109.

29. Stewart JE, Thomas KA, Lawrence CB, Dang H, Pryor BM, Peever TL: Signatures of recombination in clonal lineages of the citrus brown spot pathogen, Alternaria alternata sensu lato. Phytopathology 2013, 103:741-749.

30. Pierce NB: Black rot of oranges. Bot Gazelle 1902, 33:234-23.

31. Neergaard P: Danish Species of Alternaria and Stemphylium. London: Oxford University Press; 1945:560.

32. Ellis MB: Dematiaceous Hyphomycetes. Kew: Commonwealth Mycological Institute:19.

33. Kusaba M, Tsuge T: Nuclear ribosomal DNA variation and pathogenic specialization in Alternaria fungi known to produce host-specific toxins. Appl Environ Microbiol 1994, 60:3055-3062.

34. Simmons EG: Alternaria themes and variations (226-235): Classification of citrus pathogens. Mycotaxon 1999, 70:263-323.

35. Peever TL, Ibañez A, Akimitsu K, Timmer LW: Worldwide Phylogeography of the citrus brown spot pathogen, Alternaria alternata. Phytopathology 2002, 92:794-802.

36. Peever TL, Su G, Carpenter-Boggs L, Timmer LW: Molecular systematics of citrus-associated Alternaria spp. Mycologia 2004, 96:119-134

37. Kingman JFC: The coalescent. Stoch Process App/ 1982, 13:235-248.

38. Peever TL, Canihos Y, Olsen L, Ibanez A, Liu Y-C, Timmer LW: Population genetic structure and host specificity of Alternaria spp. causing brown spot on Minneola tangelo and rough lemon in Florida. Phytopathology 1999, 92:794-802

39. Isshiki A, Akimitsu K, Yamamoto M, Yamamoto H: Endopolygalacturonase is essential for citrus black rot caused by Alternaria citri but not brown spot caused by Alternaria alternata. Mol Plant-Microbe Interact 2001, 14:749-757.

40. Peever TL, Carpenter-Boggs L, Timmer LW, Carris LM, Bhatia A: Citrus black rot is caused by phylogenetically distinct lineages of Alternaria alternata. Phytopathology 2005, 95:512-518.

41. Su G, Peever TL, Timmer LW: Molecular systematics of citrus-associated Alternaria sp. Phytopathology 2001, 91:S190.

42. Andrew M: Molecular systematics of small-spored Alternaria species. MS Thesis: Washington State University; 2006.

43. Tran-Dinh N, Hocking A: Isolation and characterization of polymorphic microsatellite markers for Alternaria alternata. Mol Ecol Resour 2006, 6:405-407. 
44. Rozen S, Skaletsky H, Krawetz S, Misener S: Primer3 on the WWW for general users and for biologist programmers. In bioinformatics Methods and Protocols. 2000:365-386.

45. Guindon S, Gascuel O: A simple, fast, and accurate algorithm to estimate large phylogenies by maximum likelihood. Syst Biol 2003, 52:696-707.

46. Huelsenbeck JP, Ronquist F: MrBayes: Bayesian inference of phylogenetic trees. Bioinformatics 2001, 17:754-755.

47. Minin $V$, Abdo $Z$, Joyce $P$, Sullivan J: Performance-based selection of likelihood models for phylogeny estimation. Syst Biol 2003, 52:674-683.

48. Heled J, Drummond AJ: Bayesian inference of species trees from multilocus data. Mol Biol Evol 2010, 2010(27):570-580.

49. Shimodaira $H$, Hasegawa M: Multiple comparisons of loglikelihoods with applications to phylogenetic inference. Mol Biol Evol 1999, 16:1114-1116.

50. Swofford DL: PAUP*. Phylogenetic Analysis Using Parsimony ( ${ }^{*}$ and Other Methods). Version 4. Sunderland, Massachusetts: Sinauer Associates; 2003.

51. Hall TA: BioEdit: a user-friendly biological sequence alignment editor and analysis. Nucleic Acids Symp Ser 1999, 41:95-98.

52. Librado P, Rozas J: DnaSP v5: A software for comprehensive analysis of DNA polymorphism. Bioinformatics 2009, 25:1451-1452.

53. Rozas J, Sanchez-Del J, Barrio JC, Messeguer X, Rozas R: DnaSP, DNA polymorphism analyses by the coalescent and other methods. Bioinformatics 2003, 19:2496-2497.

54. Hudson RR, Kaplan NL: Statistical properties of the number of recombination events in the history of a sample of DNA sequences. Genetics 1985, 111:147-164.

55. Jakobsen IB, Easteal S: A program for calculating and displaying compatibility matrices as an aid in determining reticulate evolution in molecular sequences. Comput Appl Biosci 1996, 12:291-295.

56. Price EW, Carbone I: SNAP: workbench management tool for evolutionary population genetics analysis. Bioinformatics 2005, 21:402-404.

57. Aylor DL, Price EW, Carbone I: SNAP: combine and map modules for multilocus population genetics analysis. Bioinformatics 2006, 22:1399-1401.

58. Hey J, Wakely J: A coalescent estimator of the population recombination rate. Genetics 1997, 145:833-846.

59. Griffiths RC, Marjoram P: Ancestral inference from samples of DNA sequences with recombination. J Comput Biol 1996, 3:479-502.

60. Lawrence DP, Gannibal PB, Peever TL, Pryor BM: The sections of Alternaria: formalizing species-group concepts. Mycologia 2013, 105:530-546.

61. Bahlo M, Griffiths RC: Inference from gene trees in a subdivided population. Theor Popul Biol 2000, 57:79-95.

62. Griffiths RC, Tavaré S: Ancestral inference in population genetics. Stat Sci 1994, 9:307-319

63. Lyngso RB, Song YS, Hein J: Minimum Recombination Histories by Branch and Bound. In Algorithms in Bioinformatics, Proceedings. Berlin: Springer-Verlag Berlin; 2005:239-250.

64. Oliver JC: AUGIST: inferring species trees while accommodating gene tree uncertainty. Bioinformatics 2008, 24:2932-2933.

65. Avise JC, Ball RM Jr: Principles of genealogical concordance in species concepts and biological taxonomy. Oxf Surv Evol Biol 1990, 7:45-67.

66. Alfaro ME, Zoller S, Lutzoni F: Bayes or bootstrap? A simulation studying comparing the performance of Bayseian markov chain Monte Carlo sampling and bootstrapping in assessing phylogenetic confidence. $\mathrm{Mol}$ Biol Evol 2003, 20:255-266.

67. Maddison WP, Maddison DR: Mesquite: a modular system for evolutionary analysis. Version 2.73. 2010. http://mesquiteproject.org.

68. Yule GU: A mathematical theory of evolution, based on the conclusions of Dr. J.C. Willis, F.R.S. Philos Trans R Soc Lond Biol 1925, 213:21-87.

69. Rannala B, Yang Z: Probability distribution of molecular evolutionary tree: a new methods for phylogenetic inference. J Mol Evol 1996, 43:304-311

70. Rambaut A: FigTree, a graphical viewer of phylogenetic trees. 2008. http://tree.bio.ed.ac.uk/software/figtree.

71. Berbee ML, Payne BP, Zhang G, Roberts RG, Turgeon BG: Shared ITS DNA substitutions in isolates of opposite mating type reveal a recombining history for three presumed asexual species in the filamentous ascomycete genus Alternaria. Mycol Res 2003, 107:169-182.

72. Kasuga T, White TJ, Taylor JW: Estimation of nucleotide substitution rates in Eurotiomycete fungi. Mol Biol Evol 2002, 19:2318-2324.

73. Nishimura S, Kohmoto K: Host-specific toxins and chemical structures from Alternaria species. Annu Rev Phytopathol 1983, 21:87-116.
74. Chaverri P, Castlebury LA, Samuels GJ, Geiser DM: Multilocus phogenetics structure within the Trichoderma harzianum/Hypocrea lixii complex. Mol Phylogenet Evol 2003, 27:302-313.

75. Miller AN, Huhndorf SM: Using phylogenetic species recognition to delimit species boundaries and species relationships within Lasiosphaeria. Mycologia 2004, 96:302-313.

76. Kohmoto K, Otani H, Tsuge T: Alternaria alternata Pathogens. In Pathogenesis and Host Specificity in Plant Diss: Histopathological, Biochemical, Genetic and Molecular Bases. Vol. II, Eukaryotes. Edited by Kohmoto K, Singh US, Singh RP. Oxford: Pergamon Press; 1995:51-63.

77. Timmer LW, Peever TL, Solel Z, Akimitsu K: Alternaria diseases of citrus - Novel pathosystems. Phytopathol Mediterrian 2003, 42:3-16.

78. Nishimura S, Kohmoto K, Otani H, Ramachandran P, Tamura F: Pathological and epidemiological aspects of Alternaria alternata infection depending on a host-specific toxin. In Plant infection: the Physiological and Biochemical Basis. Edited by Asada Y, Bushnell WR, Ouchi S, Vance CP. Tokyo; Berlin: Japan Scientific Societies Press; Springer-Verlag; 1982:199-21.

79. Akamatsu H, Taga M, Kodama M, Johnson R, Otani H, Kohmoto K: Molecular karyotypes for Alternaria plant pathogens known to produce host-specific toxin. Curr Genet 1999, 35:647-656.

80. Masunaka A, Ohtani K, Peever TL, Timmer LW, Tsuge T, Yamamoto M, Akimitsu K: An isolate of Alternaria alternata that is pathogenic to both tanginess and rough lemon produces two host-selective toxins ACT and ACR toxins. Phytopathology 2005, 95:241-247.

81. Masunaka A, Tanaka A, Tsuge T, Peever TL, Timmer LW, Yamamoto M, Yamamoto H, Akimitsu K: Distribution and characterization of AKT homologs in the tangerine pathotype of Alternaria alternata. Phytopathology 2000, 90:762-768.

82. Tsuge T, Harimoto Y, Akimitsu K, Ohtani K, Kodama M, Akagi Y, Mayumi E, Yamamoto M, Otani $\mathrm{H}$ : Host-selective toxins produced by the plant pathogenic fungus Alternaria alternata. FEMS Microbiol Rev 2013, 37:44-66.

83. Li W-H, Tanimura M, Sharp PM: An evaluation of the molecular clock hypothesis using mammalian DNA sequences. J Mol Evol 1987, 25:330-342.

84. Scora RW: On the history and origin of citrus. Torrey Bot Club 1975, 102:369-375.

85. O'Donnell K: Molecular phylogeny of the Nectria haematococca-Fusarium solani species complex. Mycologia 2000, 92:919-938.

86. O'Donnell K, Kistler HC, Tacke BK, Casper HH: Gene genealogies reveal global phylogeographic structure and reproductive isolation among lineages of Fusarium graminearum, the fungus causing wheat scab. Proc Natl Acad Sci U S A 2000, 97:7905-7910.

87. Steenkamp ET, Wingfield BD, Desjardins AE: Cryptic speciation in Fusarium subglutinans. Mycologia 2002, 94:1032-1043.

88. Koufopanou V, Burt A, Taylor JW: Concordance of gene genealogies reveals reproductive isolation in the pathogenic fungus Coccidioides immitis. Proc Natl Acad Sci U S A 1997, 94:5478-5482.

89. Kasuga T, Taylor JW, White TJ: Phylogenetic relationships of varieties and geographical groups of the human pathogenic fungus, Histoplasma capsulatum Darling. J Clin Microbiol 1999, 37:653-663.

90. Cruse M, Telerant R, Gallagher T, Lee T, Taylor JW: Cryptic species in Stachybotrys chartarum. Mycologia 2002, 94:814-822.

91. Fisher MC, Koenig G, White TJ, Taylor JW: Molecular and phenotypic description of Coccidioides posadasii sp. nov., previously recognized as the non-California population of Coccidioides immitis. Mycologia 2002, 94:73-84.

92. Couch BC, Kohn LM: A multilocus gene genealogy concordant with host preference indicates segregation of a new species, Magnaporthe oryzae, from M. grisea. Mycologia 2002, 94:683-693.

93. O'Donnell K, Ward TJ, Geiser DM, Kistler HC, Aoki T: Genealogical concordance between the mating type locus and seven other nuclear genes supports formal recognition of nine phylogenetically distinct species within the Fusarium graminearum clade. Fungal Genet Biol 2004, 41:600-623.

94. Degnan JH, Rosenberg NA: Discordance of species trees with their most likely gene trees. PLoS Genet 2006, 2:762-768.

95. Rosenberg NA, Tao R: Discordance of species trees with their most likely gene trees: the case of five taxa. Syst Biol 2008, 57:131-140.

96. Sen D, Brown CJ, Top EM, Sullivan J: Inferring the evolutionary history of IncP-1 plasmids despite incongruence among backbone gene trees. Mol Biol Evol 2013, 30:154-166.

97. Belfiore NM, Liu L, Moritz C: Multilocus phylogenetics of a rapid radiation in the genus Thomomys (Rodentia: Geomyidae). Syst Biol 2008, 57:294. 
98. Yun SH, Berbee ML, Yoder OC, Turgeon BG: Evolution of the fungal self-fertile reproductive life style from self-sterile ancestors. Proc Natl Acad Sci U S A 1999, 96:5592-5597.

99. Stewart JE, Kawabe M, Abdo Z, Arie T, Peever TL: Contrasting Codon usage patterns and purifying selection at the mating locus in putatively asexual Alternaria fungal species. PLoS ONE 2011, 6:e20083.

100. Roberts RG: Alternaria yaliinficiens sp. nov. on Ya Li pear fruit: from interception to identification. Plant Dis 2005, 89:134-145.

101. Boykin LM, Armstrong KF, Kubatko L, DeBarro P: Species delimitation and global biosecurity. Evol Bioinformatics 2012, 8:1-37.

102. Rossman AY, Palm-Hernández ME: Systematics of plant pathogenetic fungi: why it matters? Plant Dis 2008, 92:1376-1386.

103. Elena K: Alternaria brown spot of Minneola in Greece; evaluation of citrus species susceptibility. Plant Pathol Eur J Plant Pathol 2006, 115:259-262.

104. Golmohammadi M, Andrew M, Peever TL, Peres NA, Timmer LW: First report of brown spot of tangerine hybrid cultivars Minneola, page and fortune caused by Alternaria alternata in Iran. Plant Pathol 2006, 12:2005-2098.

105. Marín JE, Fernández H, Peres NA, Andrew M, Peever TL, Timmer LW: First report of Alternaria brown spot of citrus caused by Alternaria alternata in Peru. Plant Dis 2006, 90:686.

doi:10.1186/1471-2148-14-38

Cite this article as: Stewart et al:: Discord between morphological and phylogenetic species boundaries: incomplete lineage sorting and recombination results in fuzzy species boundaries in an asexual fungal pathogen. BMC Evolutionary Biology 2014 14:38.

\section{Submit your next manuscript to BioMed Central and take full advantage of:}

- Convenient online submission

- Thorough peer review

- No space constraints or color figure charges

- Immediate publication on acceptance

- Inclusion in PubMed, CAS, Scopus and Google Scholar

- Research which is freely available for redistribution 